\title{
Feasibility of intermittent back-filtrate infusion hemodiafiltration to reduce intradialytic hypotension in patients with cardiovascular instability: a pilot study
}

\author{
Yutaka Koda $^{1} \cdot$ Ikuo Aoike $^{2} \cdot$ Shin Hasegawa ${ }^{3} \cdot$ Yutaka Osawa $^{4} \cdot$ Yoichi Nakagawa $^{5}$. \\ Fumio Iwabuchi $^{6} \cdot$ Chikara Iwahashi $^{7} \cdot$ Tokuichiro Sugimoto $^{8} \cdot$ Toshihiko Kikutani $^{9}$
}

Received: 7 January 2016/Accepted: 11 April 2016/Published online: 28 April 2016

(c) The Author(s) 2016. This article is published with open access at Springerlink.com

\begin{abstract}
Background Intradialytic hypotension (IDH) is one of the major problems in performing safe hemodialysis (HD). As blood volume depletion by fluid removal is a major cause of hypotension, careful regulation of blood volume change is fundamental. This study examined the effect of intermittent back-filtrate infusion hemodiafiltration (I-HDF), which modifies infusion and ultrafiltration pattern.

Methods Purified on-line quality dialysate was intermittently infused by back filtration through the dialysis membrane with a programmed dialysis machine. A bolus of $200 \mathrm{ml}$ of dialysate was infused at $30 \mathrm{~min}$ intervals. The volume infused was offset by increasing the fluid removal over the next $30 \mathrm{~min}$ by an equivalent amount. Seventyseven hypotension-prone patients with over $20-\mathrm{mmHg}$ reduction of systolic blood pressure during dialysis or intervention-requirement of more than once a week were included in the crossover study of 4 weeks duration for
\end{abstract}

Yutaka Koda

ykodan@gmail.com

1 Koda Medical and Dialysis Clinic, 3748 Yoshida, Tsubame, Niigata 959-0264, Japan

2 Koyo Medical Clinic, Konan-ku, Niigata 950-0121, Japan

3 Kashiwazaki General Medical Center, Kashiwazaki, Niigata 945-8535, Japan

4 Niigata Rinko Hospital, Higashi-ku, Niigata 950-8725, Japan

5 Nakagawa Medical Clinic, Utsunomiya, Tochigi 321-0157, Japan

6 Iwabuchi Clinic, Utsunomiya, Tochigi 320-0811, Japan

7 Gyoda General Hospital, Gyoda, Saitama 361-0056, Japan

8 Kuki General Hospital, Kuki, Saitama 346-0021, Japan

9 Ogawa Hospital, Hiki, Saitama 355-0317, Japan each modality. In a total of 1632 sessions, the frequency of interventions, the blood pressure, and the pulse rate were documented.

Results During I-HDF, interventions for symptomatic hypotension were reduced significantly from 4.5 to 3.0 (per person-month, median) and intradialytic systolic blood pressure was $4 \mathrm{mmHg}$ higher on average. The heart rate was lower during I-HDF than HD in the later session. Older patients and those with greater interdialytic weight gain responded to I-HDF.

Conclusions I-HDF could reduce interventions for IDH. It is accompanied with the increased intradialytic blood pressure and the less tachycardia, suggesting less sympathetic stimulation occurs. Thus, I-HDF could be beneficial for some hypotension-prone patients.

UMIN registration number 000013816.

Keywords Hypotension - Intermittent back-filtrate infusion · Hemodiafiltration

\section{Introduction}

Intradialytic hypotension (IDH) requires immediate medical intervention and/or nursing care. In addition, IDH may reduce the efficacy of dialysis, considerably increase a patient's discomforts such as consciousness loss, and finally lead to cardiac dysfunction with myocardial stunning [1-3]. Consequently, IDH is considered an independent risk factor for patient survival [2,4]. The high risk of IDH, methods of how to avoid it, and how to provide countermeasures are immediate issues.

One successful countermeasure is the plasma sodium biofeedback system, which can reduce the burden of IDH [5]. Fluid management, which is the other side of sodium 
management, has always been a central part of blood pressure stability. IDH is thought to occur most often with high fluid removal. Rapid removal of large interdialytic volume gain is associated with mortality and is very uncomfortable for patients [6]. In 2013, Mineshima and Eguchi advocated a convective type of method with cyclic back-filtration infusion, naming it as intermittent-infusion hemodiafiltration (I-HDF) [7]. It is a volume-centered approach to cardiovascular instability and an anti-fouling approach for membrane during convective therapy. I-HDF utilizes on-line-quality ultrapure dialysate which is back filtered with an automated dialysis machine. A quick and regular infusion is expected to restore blood volume, blood pressure, and peripheral circulation. Additionally, a backwash prevents hemodiafilter membrane fouling, mainly caused by low molecular weight protein [7]. We conducted our study to investigate whether this method can reduce the frequency of interventions for patients with IDH.

\section{Subjects and methods}

\section{Study design and patients}

The study was designed as an open, non-controlled, prospective, crossover, multicenter trial. Eight dialysis units in Japan (Niigata, Saitama, and Tochigi prefecture) participated in the study. Approval from ethics committee of Hiro Clinic Oomori, Tokyo, was obtained on 22 April, 2014 (IRB approval number: 26020), and the study was registered to UMIN (000013816).

IDH is defined in this study as systolic blood pressure falling over $20 \mathrm{mmHg}$ from baseline, or presentation of symptoms associated with hypotension requiring any medical interventions, including nursing care $[8,9]$. These symptoms include unconsciousness, nausea, vomiting, dyspnea, chest discomfort, muscle cramps, which require interventions such as bolus infusion, leg raising (Trendelenburg position), lowering or stopping ultrafiltration rate, discontinuation of dialysis, switching to sole ultrafiltration, prescription or modification of vasoconstrictor and antihypertensive agents, and so on.

Patients who experienced these IDH complications at least once a week were included in the study. The observation period before entry was 2 weeks, in which inclusion or exclusion was determined. All patients who took part in gave written informed consent. Eligible patients were stage 5 $\mathrm{CKD}$, treated by hemodialysis thrice weekly. Dry weight was clinically and comprehensively determined based on previous history and symptoms such as dyspnea, peripheral edema and blood pressure, to the weight at which patient would remain normotensive. In all participating facilities, chest
$\mathrm{X}$-ray was regularly examined to measure cardio-thoracic ratio, which might help to consider appropriateness of dry weight. Patients were excluded if: (a) they had had a cardiovascular event within the past 3 months; (b) they had active inflammatory disease or malignancy; (c) they had systolic blood pressure exceeding $150 \mathrm{mmHg}$ during dialysis; (d) they were treated with a dialysis membrane with a surface area less than $0.8 \mathrm{~m}^{2}$ or greater than $2.2 \mathrm{~m}^{2}$; or (e) they were on beta-2 microglobulin adsorptive columns.

I-HDF is a convective type of therapy which is approved by Japanese health care regulations and the reimbursement agency as a modified type of hemodiafiltration. Although the convective volume chosen for this study was too small to meet the definition set forth by the EUDIAL group [10], the study did utilize the high-flux hemodiafilter with internal filtration and intentional infusion of back-filtered ultrapure dialysate in a closed system. As the subjects were hypotension-prone in this study, intermittent infusion was expected to be prophylactic against hypotension. Dialysate was infused rapidly by backward filtration at a rate of $150 \mathrm{~mL} / \mathrm{min}$ at $30 \mathrm{~min}$ intervals using a programmable system for an automated dialysis machine, either the TR$3000 \mathrm{MA}^{\circledR}$ or the TR-3300M ${ }^{\circledR}$ (Toray Medical Ltd, Tokyo, Japan). To ensure patients safety during I-HDF the dialysate purity of the on-line fluid must be guaranteed by multiple endotoxin-retentive filters, and the entire system should be validated by manufacturers to limit endotoxins to below the applicable clinical detection limit and bacteria to less than 10 to minus 6 quality. In this study, hemodiafilter used for I-HDF period was specified to polysulfone TDF$\mathrm{M}^{\circledR}$ (Toray Medical Ltd, ultrafiltration rate: $46.6 \mathrm{~mL} /$ $0.13 \mathrm{kPa} / \mathrm{h}$ with membrane area $1.5 \mathrm{~m}^{2}$ ), and the membrane area was adjusted to limit any difference less than $0.1 \mathrm{~m}^{2}$ from the subject's prior dialyzer.

As an example, in patients with 4-h sessions, each infusion bolus was $200 \mathrm{~mL}$ and seven infusions were given per session, providing a total of $1400 \mathrm{~mL} / \mathrm{session}$ according to the protocol (see Fig. 1). Usual ultrafiltration rate based on interdialytic weight gain (IDWG) and food/water intake during session was corrected by the following equations in IHDF (corrected ultrafiltration rate: cUFR, $\mathrm{mL} / \mathrm{min}$ ).

Usual UFR $(\mathrm{mL} / \mathrm{min})=\{$ IDWG $(\mathrm{mL})$

$+[$ food and water intake during session $(\mathrm{mL})]\} /$

[session time (min)]

bolus infusion time $(\min )=[$ bolus infusion volume $(\mathrm{mL})] /$

[bolus infusion rate $(\mathrm{mL} / \mathrm{min})$ ]

corrected UFR $(\mathrm{mL} / \mathrm{min})=\{[$ usual UFR $(\mathrm{mL} / \mathrm{min})]$

$\times[$ interval time $(\mathrm{min})]+[$ bolus infusion volume $(\mathrm{mL})]\} /$

$\{[$ interval time $(\mathrm{min})]-[$ bolus infusion time $(\mathrm{min})]\}$ 
Fig. 1 Dialysate was infused by back filtration through hemodiafilter seven times in one session at a rate of $200 \mathrm{~mL} / 80 \mathrm{~s}$. The procedure was performed automatically by a programmable dialysis system

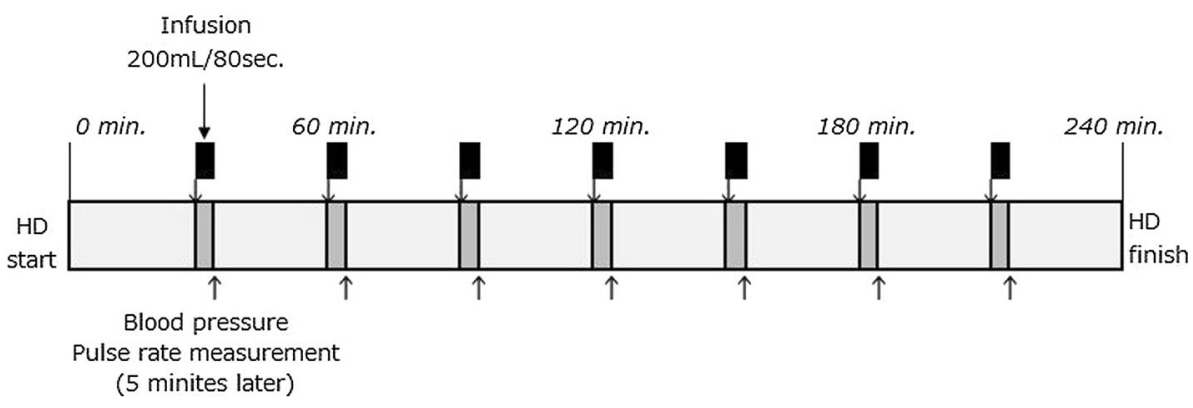

In this study, bolus infusion volume is $200 \mathrm{~mL}$, bolus infusion rate is $150 \mathrm{~mL} / \mathrm{min}$, session time is $240 \mathrm{~min}$, and interval time is $30 \mathrm{~min}$.

Crossover treatment of 2 weeks of HD, 4 weeks of I-HDF, and 2 weeks of HD was conducted. Blood pressure and pulse rate was measured at $5 \mathrm{~min}$ after each infusion (see Fig. 1). The systolic blood pressure (SBP) of these were averaged and assumed as intradialytic SBP. The total number of interventions for IDH, the blood pressure, and the pulse rate were recorded in the last week of each study period. Data from the two HD periods (pre and post I-HDF treatment) for each patient were combined and averaged, and served as a single control.

The protocol indicated that dialysis parameters and medications related to blood pressure were to be kept the same during both treatments for the purpose of this study. However, it was allowed to change them in accordance with clinical state. If any changes were made, they were documented as interventions. The primary outcome of this study was the frequency of interventions for IDH. An intervention-decreased patient in I-HDF period is considered as an effective and improved case and defined as responder for I-HDF.

\section{Statistical evaluation}

Results were presented as mean $\pm \mathrm{SD}$, median and interquartile range or IQR: 25th, 75th percentile, depending on variable distributions, and as percent for categorical variables. Analyses were conducted by Chi-squared test, Student's $t$ test (paired or non-paired), and Wilcoxon singed-rank test, where appropriate. Multivariate logistic regressions were applied to examine predictors for patients who responded to I-HDF.

All of the analyses were conducted using $\mathrm{R}$ version 3.1.2 software for statistical computing ( $\mathrm{R}$ Foundation for Statistical Computing, Vienna, Austria: http://www.R-project. org/).

\section{Results}

\section{Total entry and dropouts}

A total of 77 patients were enrolled in the study. Table 1 summarizes the frequency and cause of dropouts. Nine patients dropped out. Six patients among them were included in the final analysis as worsened cases, though they were not necessarily exacerbated. Remaining 3 patients were not included because they withdrew before I-HDF. Thus, 68 patients completed the study and 74 patients were in final analysis.

The details of dropouts are summarized in Table $1 \mathrm{~b}$. The characteristics of 9 dropouts, compared with 68 completed cases (dropouts vs. completed) were older age (76.2 \pm 8.4 vs. $66.1 \pm 11.2$ years old, $P=0.003)$ and shorter session time $(213.3 \pm 31.6$ vs. $234.3 \pm 18.1 \mathrm{~min}$, $P=0.002)$. Other demographics were not different.

\section{Baseline demographics and dialysis parameters}

Baseline demographics and dialysis parameters of patients in the final analysis are summarized in Table 2.

\section{Primary outcome}

Table 3 summarizes the data from the I-HDF periods compared to the HD data, including the primary outcomes in patients who completed the study. A total of 819 interventions were registered during 816 sessions of HD. The total interventions reduced from 819 to 668 during I-HDF ( $-18.4 \%$, see Fig. 2), from a median frequency of 4.5 to 3.0 times per person (Wilcoxon signed-rank test, $P=0.003$, see Fig. 3). Details of interventions during the each 4-weeks period (frequency, HD vs. I-HDF) were bolus infusion (27 vs. 24), leg raising with Trendelenburg position (181 vs. 129), lowering ultrafiltration rate (82 vs. 54), switching to sole ultrafiltration (11 vs. 14), discontinuation of session (9 vs. 9), prescription or modification 
Table 1 Total entry and dropouts

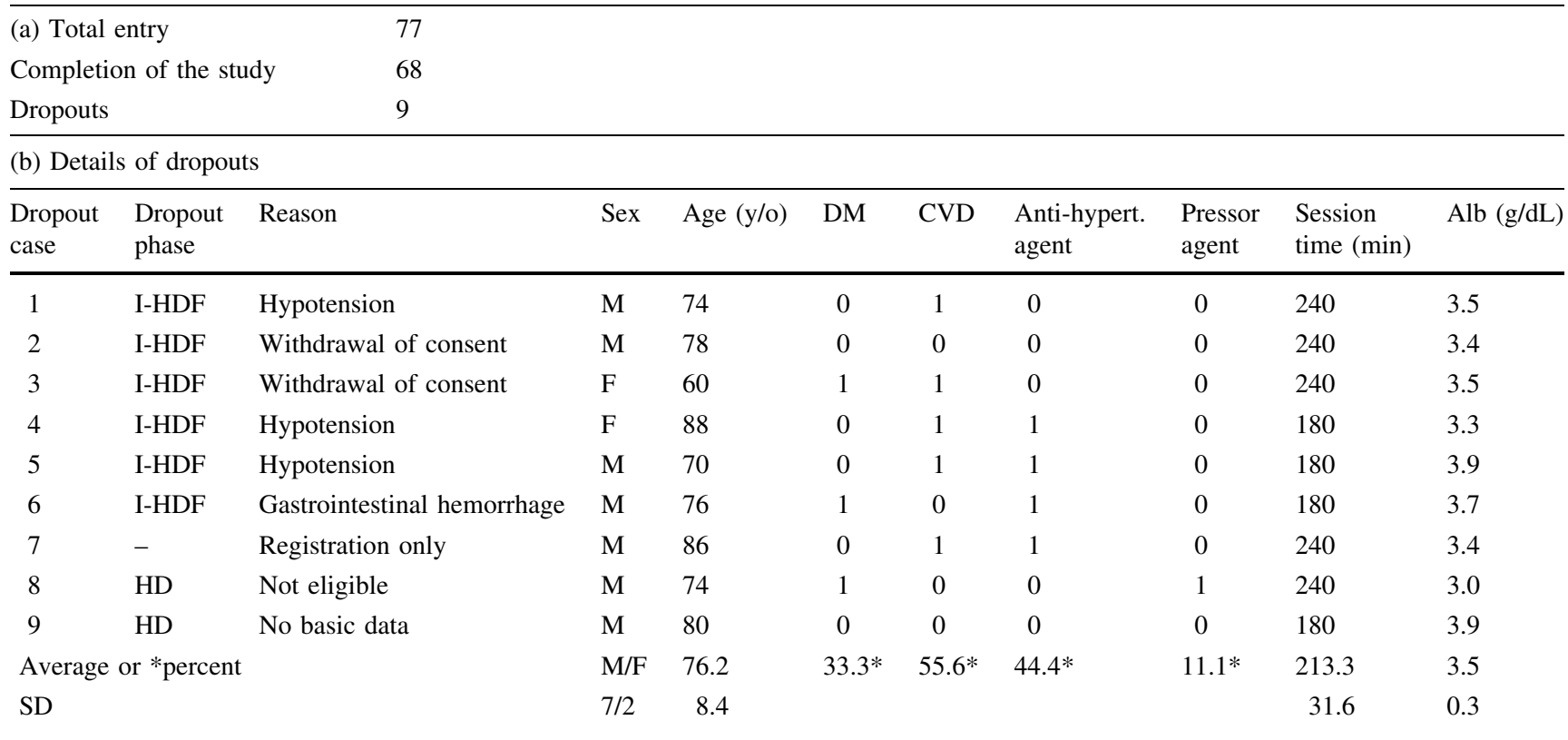

In the columns of DM, CVD, antihypertensive agent and pressor agent, the presence was indicated by 1 , and the absence by 0 . Cases $1-6$ were included as worsened in the final analysis

$D M$ diabetes mellitus, $C V D$ cardiovascular disease, Anti-hypert. agent anti-hypertensive agent, Alb albumin, y/o years old, $S D$ standard deviation

Table 2 Patient demographics and dialysis parameters (final analysis including 6 dropouts)

\begin{tabular}{|c|c|c|}
\hline & $n=74$ & Range \\
\hline \multicolumn{3}{|l|}{ Patient demographics } \\
\hline Age (years) & $66.7 \pm 11.3$ & $35-87$ \\
\hline Male $(\%)$ & 52.7 & \\
\hline Diabetics $(\%)$ & 32.4 & \\
\hline Vintage (years; median [IQR]) & $4.0[2.0-10.0]$ & $0.3-28.0$ \\
\hline Dry weight $(\mathrm{kg})$ & $54.7 \pm 10.9$ & $37.4-82.5$ \\
\hline Interdialytic weight gain (\% of DW) & $4.3 \pm 1.3$ & $1.7-7.3$ \\
\hline Serum albumin $(\mathrm{g} / \mathrm{dL})$ & $3.6 \pm 0.4$ & $2.8-4.5$ \\
\hline Cardiovascular disease $(\%)$ & 44.5 & \\
\hline Antihypertensive agent (\%) & 50.0 & \\
\hline Pressor agent $(\%)$ & 24.3 & \\
\hline \multicolumn{3}{|l|}{ Dialysis parameters } \\
\hline Blood flow (mL/min) & $200[200-200]$ & $160-230$ \\
\hline Dialysate flow (mL/min; median [IQR]) & $500[500-500]$ & $500-600$ \\
\hline Session time (min; median [IQR]) & $240[240-240]$ & $180-270$ \\
\hline Dialysate Sodium (mEq/L; median [IQR]) & $140[140-140]$ & $140-143$ \\
\hline Dialysate temperature $\left({ }^{\circ} \mathrm{C}\right.$; median $\left.[\mathrm{IQR}]\right)$ & $36[36.0-36.5]$ & $36.0-36.5$ \\
\hline Dialysate buffer, bicarbonate-based; citrate/acetate & $32 / 42$ & \\
\hline
\end{tabular}

Values are given in mean $\pm \mathrm{SD}$ for normally distributed variables, in median [IQR interquartile range] for non-normally distributed and \% for categorical variables

$D W$ dry weight

of vasoconstrictor and antihypertensive agents (318 vs. 271) and others (191 vs. 167).

Along with this, hypotensive sessions also reduced from 337 in HD to 298 in I-HDF $(P=0.021)$. Intradialytic SBP obtained $5 \mathrm{~min}$ after infusion increased by $4 \mathrm{mmHg}$ in average ( $P=0.006$, see Fig. 3$)$, in spite of the absence of any change in pre- and post-SBP. Dry weight, pre- and post-body weight did not change throughout the study 
Table 3 Interventions, blood pressure and laboratory data in cross-over treatment completed cases
Total interventions

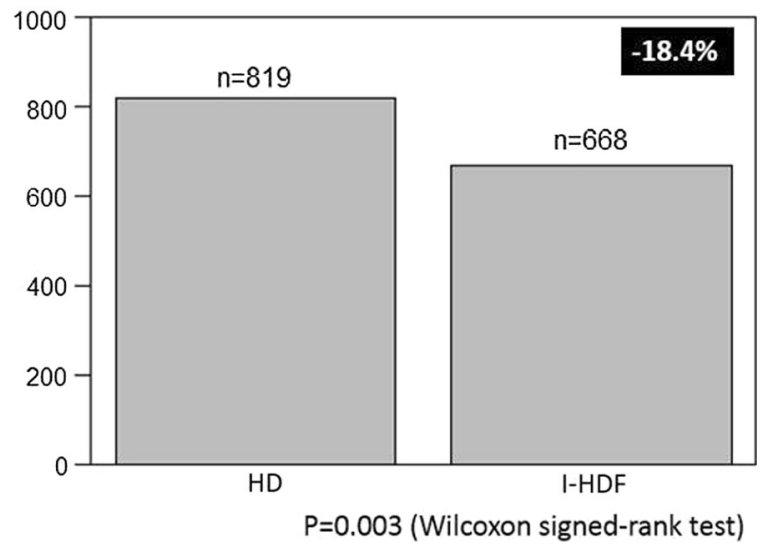

Fig. 2 Total number of IDH-related medical and nursing interventions in both HD and I-HDF periods (/816 sessions). $P$ value was obtained by the comparison of paired data using Wilcoxon signedrank test

\begin{tabular}{|c|c|c|c|c|}
\hline Parameter & $\mathrm{HD}(n=68)$ & $\mathrm{I}-\mathrm{HDF}(n=68)$ & $P$ value & Statistics \\
\hline No. of sessions & 816 & 816 & & \\
\hline No. of interventions & 819 & 668 & 0.003 & *, \#\#\# \\
\hline Interventions per person (median [IQR]) & $4.5[0.8-20.3]$ & $3.0[0-13.3]$ & 0.003 & $*$, \#\#\# \\
\hline No. of sessions with hypotension & 337 & 298 & 0.021 & *, \#\#\# \\
\hline Predialysis SBP (mmHg) & $134.6 \pm 20.8$ & $135.3 \pm 23.0$ & 0.774 & \# \\
\hline Intradialytic SBP (mmHg) & $125.7 \pm 18.6$ & $129.7 \pm 19.8$ & 0.006 & $*, \#$ \\
\hline Postdialysis SBP (mmHg) & $130.0 \pm 25.2$ & $131.2 \pm 26.3$ & 0.592 & $\#$ \\
\hline DW (kg) & $54.9 \pm 10.8$ & $54.9 \pm 10.8$ & 0.852 & $\#$ \\
\hline Predialysis weight (kg) & $57.0 \pm 11.1$ & $57.1 \pm 11.2$ & 0.534 & $\#$ \\
\hline Postdialysis weight (kg) & $55.0 \pm 10.7$ & $54.9 \pm 10.7$ & 0.369 & $\#$ \\
\hline Predialysis PR (beats/min) & $75.1 \pm 12.5$ & $76.1 \pm 12.3$ & 0.628 & $\#$ \\
\hline Intradialytic PR (beats/min, at $185 \mathrm{~min}$ ) & $73.1 \pm 12.8$ & $70.6 \pm 13.9$ & 0.007 & $*, \#$ \\
\hline Postdialysis PR (beats/min) & $72.4 \pm 13.2$ & $71.8 \pm 14.5$ & 0.39 & $\#$ \\
\hline Net UF (L/session) & $2.3 \pm 0.7$ & $2.4 \pm 0.7$ & 0.588 & $\#$ \\
\hline Interdialytic weight gain (\% of DW) & $4.3 \pm 1.3$ & $4.4 \pm 1.3$ & 0.597 & $\#$ \\
\hline Total back-filtrate infusion (mL/session) & 0 & $1362 \pm 121$ & $p<0.001$ & $*, \#$ \\
\hline UFR in HD, cUFR in I-HDF $(\mathrm{mL} / \mathrm{h} / \mathrm{kg})$ & $10.3 \pm 3.1$ & $19.5 \pm 4.2$ & $p<0.001$ & $*, \#$ \\
\hline Membrane area $\left(\mathrm{m}^{2}\right)$ & $1.68 \pm 0.28$ & $1.62 \pm 0.27$ & 0.001 & $*, \#$ \\
\hline Membrane material (PS, \%) & 82.4 & 100 & $p<0.001$ & $*, \#$ \\
\hline Hematocrit (\%) & $32.9 \pm 3.2$ & $32.4 \pm 3.6$ & 0.088 & \\
\hline BUN (mg/dL) & $60.6 \pm 13.2$ & $61.0 \pm 12.5$ & 0.845 & \\
\hline Urea reduction ratio $(\%)$ & $70.7 \pm 7.9$ & $68.3 \pm 6.2$ & $p<0.001$ & $*, \#$ \\
\hline
\end{tabular}

Values are given in mean \pm SD in normally distributed, median [IQR interquartile range] in non-normally distributed and \% in categorical variables

$D W$ dry weight, $P S$ polysulfone, $S B P$ systolic blood pressure, $P R$ pulse rate, $U F R$ ultrafiltration rate, $B U N$ blood urea nitrogen, $c U F R$ corrected UFR in I-HDF (see text)

\#\#\# Wilcoxon signed-rank test, ${ }^{\# \#}$ Chi-square test, ${ }^{\#}$ paired $t$-test, * statistically significant 

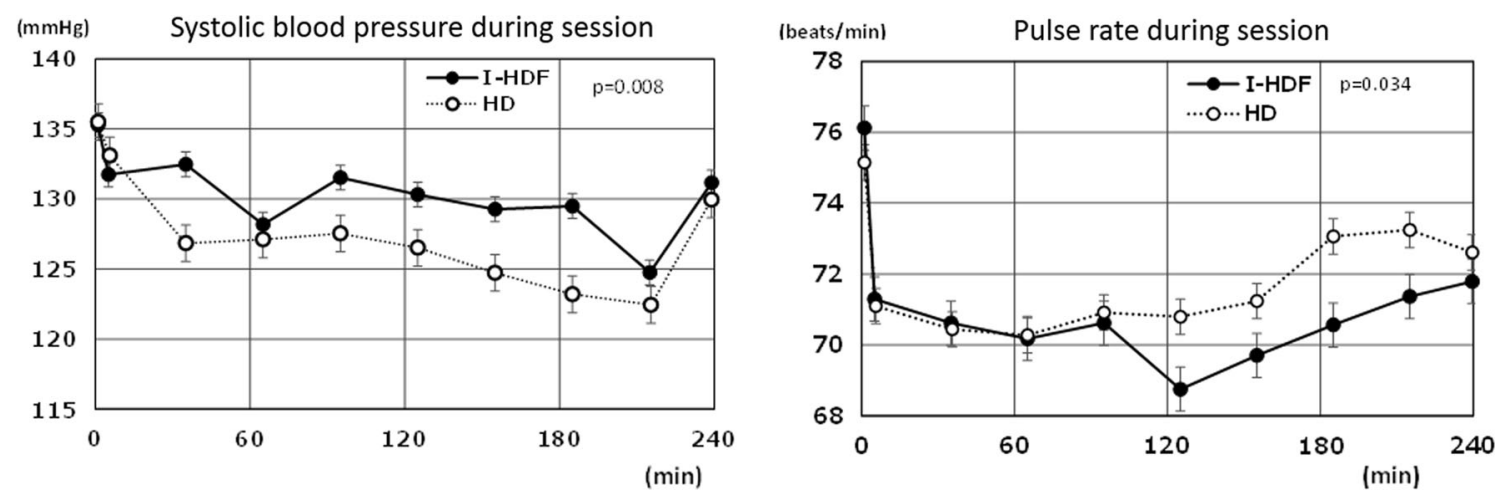

Fig. 4 Systolic blood pressure and pulse rate change in HD and I-HDF session; $P$ value for the comparison of HD and I-HDF using ANOVA

Table 4 Characteristics of responders and non-responders to I-HDF

\begin{tabular}{|c|c|c|c|c|}
\hline & Responders $(n=34)$ & Non-responders $(n=34)$ & $P$ value & Statistics \\
\hline Delta frequency of intervention $[\mathrm{IQR}]^{\mathrm{a}}$ & $-4[-8,-2]$ & $1[0,1]$ & & \\
\hline Age (years) & $68.6 \pm 10.7$ & $63.6 \pm 11.3$ & 0.066 & \# \\
\hline Male $(\%)$ & 50.0 & 52.9 & 0.808 & $\#$ \\
\hline Diabetics $(\%)$ & 38.2 & 26.4 & 0.299 & \#\# \\
\hline Vintage (years; median [IQR]) & $4.0[2.0-8.8]$ & $4.7[1.9-12.8]$ & 0.605 & \#\#\# \\
\hline Dry weight $(\mathrm{kg})$ & $53.8 \pm 9.3$ & $55.6 \pm 12.3$ & 0.479 & \# \\
\hline Serum albumin $(\mathrm{g} / \mathrm{dL})$ & $3.55 \pm 0.41$ & $3.62 \pm 0.36$ & 0.453 & $\#$ \\
\hline Cardiovascular disease $(\%)$ & 47.1 & 35.3 & 0.324 & \#\# \\
\hline Antihypertensive agent (\%) & 47.1 & 52.9 & 0.628 & \#\# \\
\hline Pressor agent $(\%)$ & 38.2 & 14.7 & 0.028 & $*$, \#\# \\
\hline Predialysis SBP $(\mathrm{mmHg})$ & $136.2 \pm 22.8$ & $134.4 \pm 23.7$ & 0.751 & $\#$ \\
\hline Intradialytic SBP increment $(\mathrm{mmHg})$ & $6.9 \pm 12.4$ & $0.9 \pm 9.8$ & 0.029 & $*$, \# \\
\hline Pulse rate at $35 \mathrm{~min}$ (beats/min) & $70.3 \pm 14.4$ & $71.0 \pm 11.0$ & 0.821 & $\#$ \\
\hline Net UF (L/session) & $2.46 \pm 0.78$ & $2.17 \pm 0.68$ & 0.106 & $\#$ \\
\hline cUFR $(\mathrm{mL} / \mathrm{h} / \mathrm{kg})$ & $20.6 \pm 3.4$ & $18.3 \pm 4.6$ & 0.032 & $*$, \# \\
\hline Interdialytic weight gain ( $\%$ of DW) & $4.8 \pm 1.1$ & $4.0 \pm 1.5$ & 0.017 & $*$, \# \\
\hline Membrane area $\left(\mathrm{m}^{2}\right)$ & $1.69 \pm 0.28$ & $1.67 \pm 0.29$ & 0.730 & $\#$ \\
\hline Hematocrit $(\%)$ & $32.6 \pm 4.1$ & $32.2 \pm 3.0$ & 0.599 & $\#$ \\
\hline BUN (mg/dL) & $59.6 \pm 12.2$ & $62.3 \pm \pm 12.8$ & 0.382 & $\#$ \\
\hline Urea reduction ratio $(\%)$ & $71.9 \pm 5.0$ & $69.5 \pm 7.1$ & 0.123 & \# \\
\hline Blood flow (mL/min) & $200[200-200]$ & $200[200-200]$ & 0.987 & \#\#\# \\
\hline Session time (min) & $239.1 \pm 11.6$ & $229.4 \pm 22.0$ & 0.017 & $*$, \# \\
\hline Dialysate buffer (citrate/acetate) & $17 / 17$ & $15 / 19$ & 0.808 & \#\# \\
\hline
\end{tabular}

Values are given in mean \pm SD in normally distributed, median [IQR interquartile range] in non-normally distributed and $\%$ for categorical variables

$B V$ blood volume, $c U F R$ corrected UFR in I-HDF (see text)

\#\#\# Wilcoxon signed-test, ${ }^{\# \#}$ Chi-square test, ${ }^{\#} t$-test, ${ }^{*}$ statistically significant

${ }^{\text {a }}$ (Interventions during I-HDF) - (interventions during HD)

period. Very importantly, intradialytic pulse rate was lower in I-HDF (see Fig. $4, P=0.007$ ). Membrane area and material were significantly different, but this was due to protocol compliance.
The data were reanalyzed finally with inclusion of data from the six patients who dropped out during the I-HDF period after assigning these subjects the median intervention score of the worsened subjects. In this final analysis 
Table 5 Predictors associated with intervention-reduction by multiple logistic regression analysis

\begin{tabular}{lcccc}
\hline Parameter & Estimate & $P$-value & OR & 95 \% CI of OR \\
\hline Age (years) & 0.080 & 0.016 & 1.083 & $1.019-1.161^{*}$ \\
Interdialytic weight gain (\%) & 0.641 & 0.018 & 1.899 & $1.162-3.401^{*}$ \\
Pressor agent & 1.262 & 0.090 & 3.531 & $0.885-17.31$ \\
Session time (h) & 1.970 & 0.143 & 7.169 & $0.640-189.2$ \\
Hemodialysis vintage (years) & -0.075 & 0.149 & 0.928 & $0.827-1.019$ \\
History of CVD & -0.564 & 0.407 & 0.569 & $0.138-2.065$ \\
Diabetics & -0.338 & 0.634 & 0.713 & $0.171-2.873$ \\
\hline
\end{tabular}

We can say that 1 year increase in age, the odds of intervention-reduction by I-HDF (vs. not reduced) increase by a factor of 1.083 and $1 \%$ increase in IDWG (\% of dry weight), the odds increase by a factor of 1.899

Likelihood ratio test, $P=0.005$

$C V D$ cardiovascular disease, $O R$ odds ratio, $C I$ confidence interval

* Statistically significant the reduction in intervention frequency remained significant $(P=0.041)$.

\section{Predictive factor for I-HDF responder}

To elucidate the characteristics of improved cases for I-HDF, the patients with reduced intervention frequency were treated as responders $(n=34)$, and the patients with the same or increased frequency as non-responders $(n=34)$ (see Table 4).

In univariate comparative analysis, intradialytic SBP rise during $\mathrm{HD}$ was $6 \mathrm{mmHg}$ greater in responders. The decrease in interventions thus was paralleled by the observation of greater blood pressure elevation. The treatment time, IDWG and the pressor agent prescription were significantly different between the groups. The corrected UFR was unexpectedly greater in responders. Although not significant, age was suspected to have any effect $(P=0.066)$.

Based on the above result, multiple logistic regression analysis with 7 variables was performed. In addition to the variables having a low $P$ value in the univariate comparative analysis, the variables we wish to know its clinical association were selected. The analysis revealed that patients who were older and those with greater IDWG had significant benefit (see Table 5).

\section{Discussion}

IDH is very common, occurring in $20-30 \%$ in chronic hemodialysis patients [1,9]. Recently, attention has centered on whether IDH can cause organ ischemic damage, such as to the heart muscle and brain [11]. If IDH is repetitive, it impairs a patient's quality of life, adequate dialysis dose, and organ/peripheral circulation, finally affecting mortality rate [4]. Although risk factors have not been clearly defined, older age, diabetes, cardiovascular illness, autonomic dysfunction, poor nutritional status, severe anemia, dialysate composition, and large IDWG (massive ultrafiltration volume) are considered predispositions for IDH [11]. Altered ET-1 levels [12] and heart rate variance [13] are also involved in the pathogenesis of hypotension during HD.

Among these risk factors, ultrafiltration leading to hypovolemia is the most essential and important factor causing IDH during hemodialysis $[1,2,6]$. Recently, much attention has been given to the convective method, which has a beneficial effect on blood pressure. Locatelli et al. reported that convective therapy reduced intradialytic hypotension, especially the use of on-line predilution HDF [14]. They speculated that the convective mode may have a positive sodium balance and also reduce core temperature which would, contribute to the results, but the true mechanism was still inconclusive.

We centered on a volume-modifying approach, namely intermittent back-filtration infusion HDF, or I-HDF. Mineshima and Eguchi first proposed the method in 2013 [7]. In addition to its anti-hypotensive effect, it improves peripheral circulation monitored by laser flow meter, and increases solute removal, probably due to volume-based vasodilation [7]. Intentional back filtration is performed by a programmed automated dialysis machine with a relatively long cycle (every $30 \mathrm{~min}$ ), thus the membrane is backwashed to recover some fouling. Low molecular weight protein clearance is maintained better by I-HDF than by conventional continuous filtration. Thus, I-HDF has two points of action: peripheral circulation improvement and membrane fouling prevention [7].

The main finding of this study is the demonstration of lower intervention frequency for IDH in hypotension-prone patients during the I-HDF period (see Figs. 2, 3). 
Interventions were reduced $-18.4 \%$ in total, 4.5-3.0 (per person-month) in median. The lower frequency was clearly associated with a significant increase of intradialytic SBP (see Tables 3, 4). Intermittent infusion irrespective of blood pressure might have mitigated asymptomatic hypotension occurring more frequently than we expected [15].

In addition, pulse rate was significantly lower in I-HDF, suggesting a less sympathetic stimulation (see Fig. 4). Here, we must emphasize that the pulse rate data indicate that I-HDF maintained sympathetic stability. Ultrafiltration increases activity of the sympathetic system and induces compensatory vasoconstriction. Vasoconstriction decreases the dermal tissue circulation and, as a result, core body temperature tends to increase, leading to a higher likelihood of acute hypotension [16]. In physiological conditions, dermal blood flow fluctuates very dynamically. Excessive arteriolar vasoconstriction mediated by sympathetic activation may stimulate the production of adenosine, an endogenous vasodilator [17], and its implication is that hypotension may develop suddenly even when blood volume is still maintained. In addition, a decrease in sympathetic stimulation should increase arteriolar blood flow exponentially [18] and thus improve solute exchange in the capillary bed which should improve final solute removal in I-HDF. Thus, weak sympathetic stimulation in I-HDF should improve cardiovascular stability, prevent ischemic organ injury, and enhance solute removal through capillary and dialysis membranes.

Anti-hypotensive effect of I-HDF was more pronounced in older patients and in patients exceeding a certain level of volume-gain, when analyzed by multiple logistic regression. This may be explained partly by the hypothesis that selected younger, hypotension-prone, patients with less weight-gain may have more complicated problems than mere volume issues, and these problems were not corrected by infusion. The possibility of confounding by unmeasured or unknown factors cannot be excluded. For example, although eating during dialysis may be an important trigger for IDH, we did not survey the relation between them in the present study.

Higher UFR was associated with greater risk of cardiovascular mortality in the hemodialysis (HEMO) study patients [6]. The corrected UFR during I-HDF increased to a range associated with increased risk in that report. However, an important difference to note is that I-HDF is characterized by intermittent infusion, namely intermittent extracellular fluid expansion and blood pressure increment.

A weakness of this study is its relatively small sample size. Although the results obtained are statistically significant, it is necessary to establish that I-HDF really improves comorbidity and survival in a greater number of patients. Further technical improvement is required, such as eventual incorporation of a biofeedback system [5, 19], and replacement volume should be standardized by body size.

In studies involving the treatment of sick people, a substantial dropout rate can be anticipated. There were nine dropouts $(11 \%)$ in this study, but we eventually included six who stopped during I-HDF as worsened cases, irrespective of the reason the individual dropped out. However, we must be cautious in interpreting the clinical results when dropouts are present in a crossover study.

A strength of the study is that the observed reduction in intervention correlates with the observation of increased blood pressure during treatment. It gives validity to the results and supports the usefulness of I-HDF as a countermeasure to IDH. The hemodiafilter membrane material was also matched during I-HDF (polysulfone). This should exclude the possibility that our results were influenced by membrane differences that occurred during I-HDF.

Extrapolating from the results of this multicenter study, we may hypothesize that modifying the infusion rate and infusion frequency during treatment of hypotension-prone patients, especially in the case of seniors (around 69 years) with modest ultrafiltration volume $(4.8 \%$ of post dialysis weight) may yield further benefits. Finally, I-HDF is economical because it requires no medications.

\section{Conclusion}

I-HDF could be a measure to reduce the need for medical intervention and nursing care in some patients with IDH, and associated with raising intradialytic systolic blood pressure while decreasing sympathetic stimulation. The results imply that the current practice of ultrafiltration during dialysis still needs refinement.

Acknowledgments The authors would like to thank the members of the advisory committee: Tetsuro Takeda (Dokkyo Medical University, Koshigaya Hospital, Saitama), Junichiro J. Kazama (Niigata University, Niigata), Noriaki Iino (Uonuma-Kikan Hospital, Niigata University, Niigata). The following collaborators also participated in this study: Keisi Furumura (Nakagawa Medical Clinic), Yoji Koizumi (Iwabuchi Clinic), Tetsuya Otani (Gyoda General Hospital), Takeshi Omi (Kuki General Hospital), Yusuke Kobayashi (Ogawa Hospital), Tatsuya Tanaka (Kashiwazaki General Medical Center Hospital), Naoki Nakamura (Niigata Rinko hospital), and Tetsuo Maruyama (Koda Medical and Dialysis Clinic). We thank all of the physicians, nurses, and dialysis technicians of the participating centers for data collection. We also thank Toray Industries, Inc. for providing financial support for the study.

\section{Compliance with ethical standards}

Conflict of interest statement This physician-led clinical trial was performed by the funding of Toray Industries, Inc. All authors have declared no competing interest in relation to this paper. 
Open Access This article is distributed under the terms of the Creative Commons Attribution 4.0 International License (http://crea tivecommons.org/licenses/by/4.0/), which permits unrestricted use, distribution, and reproduction in any medium, provided you give appropriate credit to the original author(s) and the source, provide a link to the Creative Commons license, and indicate if changes were made.

\section{References}

1. Daugirdas JT. Pathophysiology of dialysis hypotension: an update. Am J Kidney Dis. 2001;38(Suppl 4):S11-7.

2. Henderson LW. Symptomatic intradialytic hypotension and mortality: an opinionated review. Semin Dial. 2012;25:320-5.

3. Burton JO, Jefferies HJ, Selby NM, McIntyre CW. Hemodialysisinduced cardiac injury: determinants and associated outcomes. Clin J Am Soc Nephrol. 2009;4:914-20.

4. Shoji T, Tsubakihara Y, Fujii M, Imai E. Haemodialysis-associated hypotension as an independent risk factor for two-year mortality in hemodialysis patients. Kidney Int. 2004;66:1212-20.

5. Locatelli F, Stefoni S, Petitclerc T, Colì L, Filippo SD, Andrulli $\mathrm{S}$, et al. Effect of a plasma sodium biofeedback system applied to HFR on the intradialytic cardiovascular stability. Results from a randomized controlled study. Nephrol Dial Transplant. 2012;27:3935-42.

6. Flythe JE, Kimmel SE, Brunelli SM. Rapid fluid removal during dialysis is associated with cardiovascular morbidity and mortality. Kidney Int. 2011;79:250-7.

7. Mineshima M, Eguchi K. Development of intermittent infusion hemodiafiltration using ultrapure dialysis fluid with an automated dialysis machine. Blood Purif. 2013;35(Suppl 1):55-8.

8. Kooman J, Basci A, Pizzarelli F, Canaud B, Haage P, Fouque D, et al. EBPG guideline on haemodynamic instability. Nephrol Dial Transplant. 2007;22(Suppl 2):S22-44.

9. K/DOQI Workgroup. K/DOQI clinical practice guidelines for cardiovascular disease in dialysis patients. Am J Kidney Dis. 2005;45(Suppl 3):S1-153.
10. Tattersall JE, Ward RA, EUDIAL group. Online haemodiafiltration: definition, dose quantification and safety revisited. Nephrol Dial Transplant. 2013;22:542-50.

11. Davenport A. What are the causes of the ill effects of chronic hemodialysis? Balancing risks: blood pressure targets, intradialytic hypotension, and ischemic brain injury. Semin Dial. 2014;27:13-5.

12. Chang YM, Shiao CC, Chang KC, Chen IL, Yang CL, Leu SC, et al. Heart rate variability is an indicator for intradialytic hypotension among chronic hemodialysis patients. Clin Exp Nephrol. 2015;. doi:10.1007/s10157-015-1189-9 (Epub ahead of print).

13. El-Shafey EM, El-Nagar GF, Selim MF, El-Sorogy HA, Sabry AA. Is there a role for endothelin- 1 in the hemodynamic changes during hemodialysis? Clin Exp Nephrol. 2008;12(5):370-5.

14. Locatelli F, Altieri P, Andrulli S, Bolasco P, Sau G, Pedrini LA, et al. Hemofiltration and hemodiafiltration reduce intradialytic hypotension in ESRD. J Am Soc Nephrol. 2010;21:1798-807.

15. Meredith DJ, Pugh CW, Sutherland S, Tarassenko L, Birks J. The relationship between symptoms and blood pressure during maintenance hemodialysis. Hemodial Int. 2015;19(4):543-52.

16. Gotch FA, Keen ML, Yarian SR. An analysis of thermal regulation in hemodialysis with one and three compartment models. ASAIO Trans. 1989;35:622-4.

17. Shinzato T, Miwa M, Nakai S, Morita H, Odani $H$, Inoue I, Maeda K, et al. Role of adenosine in dialysis-induced hypotension. J Am Soc Nephrol. 1994;4(12):1987-94.

18. Guyton AC, Hall JE (2011) Overview of the circulation; biophysics of pressure flow, and resistance. In: Textbook of medical physiology, 12th edn. Saunders, Elsevier; Philadelphia, pp 157-166.

19. Déziel C, Bouchard J, Zellweger M, Madore F. Impact of hemocontrol on hypertension, nursing interventions, and quality of life: a randomized, controlled trial. Clin J Am Soc Nephrol. 2007;2:661-8. 